\title{
Lung sarcoidosis in children: update on disease expression and management
}

\author{
Nadia Nathan, ${ }^{1,2,3}$ Pierre Marcelo, ${ }^{4}$ Véronique Houdouin, ${ }^{5}$ Ralph Epaud, $, 6,8$ \\ Jacques de Blic, ${ }^{9,10}$ Dominique Valeyre, ${ }^{11}$ Anne Houzel, ${ }^{12}$ Pierre-François Busson, ${ }^{13}$ \\ Harriet Corvol, ${ }^{1,2,3}$ Antoine Deschildre, ${ }^{4}$ Annick Clement, ${ }^{1,2,3}$ for the RespiRare and \\ the French Sarcoidosis groups
}

For numbered affiliations see end of article.

Correspondence to Dr Nadia Nathan, Pediatric Pulmonary Department, Hôpital Armand Trousseau, 26 avenue du Dr Arnold Netter, Paris 75012, France; nadia.nathan@trs.aphp.fr

Received 22 January 2015 Revised 12 March 2015 Accepted 16 March 2015 Published Online First 8 April 2015

\section{CrossMark}

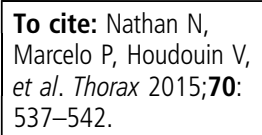

\section{ABSTRACT}

Background Sarcoidosis is a rare lung disease in children. The aim of the present study was to provide update information on disease presentation and progression, patient management and prognosis factors in a cohort of children with lung sarcoidosis.

Methods With the network of the French Reference Centre for Rare Lung Diseases (RespiRare), we collected information on a large cohort of paediatric thoracic sarcoidosis to provide information on disease presentation, management and outcome.

Results Forty-one patients were included with a median age at diagnosis of 11.8 years (1.1-15.8), mostly from Afro-Caribbean and Sub-Saharan origin. At diagnosis, $85 \%$ presented with a multi-organic disease, and no major differences were found regarding disease severity between the patients diagnosed before or after 10 years old. Corticosteroids were the most used treatment, with more intravenous pulses in the youngest patients. The 18-month outcome showed that patients diagnosed before 10 years old were more likely to recover $(50 \%$ vs $29 \%$ ), and presented fewer relapses ( $29 \%$ vs $58 \%$ ). At 4-5 years of follow-up, relapses were mostly observed for patients diagnosed after 10 years old.

Discussion In the included children, mostly of AfroCaribbean and Sub-Saharan origin, sarcoidosis seems severe, with multi-organic involvement and foreground general symptoms. Common prognosis factors are not suitable in paediatric patients, and a young age at diagnosis does not seem to be associated with a poorer prognosis. The study is ongoing to provide further information on the very-long-term follow-up of paediatric sarcoidosis.

\section{BACKGROUND}

Sarcoidosis is a chronic inflammatory disease of unknown cause, characterised by the formation of non-caseating granuloma in various organs. ${ }^{1}$ Its pathophysiology remains unclear, with, however, increasing evidence that genetic susceptibility and environmental factors contribute to disease development. ${ }^{2-5}$ It is a rare disease reported mainly in adult patients aged $25-45$ years, with an estimated incidence of 5-45 in 100000 population. ${ }^{6}$ In children, data derived from the nationwide Denmark registry provide an estimation of the overall incidence of 0.29 per 100000 children, mainly in

\section{Key messages}

\section{What is the key question?}

- Paediatric sarcoidosis is very rare; this is a national cohort study updating data on this disease providing a comparison with adult sarcoidosis and with other published cases of paediatric sarcoidosis.

\section{What is the bottom line?}

- Common prognosis factors in adults are not suitable in paediatric patients; sarcoidosis is a multi-organic severe disease in this cohort, mostly of Afro-Caribbean and Sub-Saharan origin.

\section{Why read on?}

- This study presents a large new cohort of paediatric sarcoidosis, and raises differences compared with the Danish paediatric cohort, and with adult forms of sarcoidosis in terms of presentation, management and outcome.

children older than 10 years. ${ }^{7}$ Familial cases have also been reported. ${ }^{8}$

Sarcoidosis can affect many organs, predominantly the lung. ${ }^{10}$ Disease expression depends on the location of the granuloma. ${ }^{11}$ In children, apart from the Danish and the French studies, information on sarcoidosis expression and progression remains limited to small series of patients. ${ }^{12}$ Consequently, disease management is often derived from recommendations established in adult patients.

The aim of the present study was to provide update information on disease presentation and progression in a cohort of children with lung sarcoidosis, and to discuss patient management based on disease expression and severity.

\section{MATERIALS AND METHODS}

All patients with a paediatric form of lung sarcoidosis at the time of diagnosis and registered in the database of the French reference centre for rare lung diseases RespiRare were included. As previously described, RespiRare is the national organisation for rare lung diseases in France, launched by the Ministry of Health in $2006{ }^{13}$ Since 2008 , the RespiRare database has been organised and structured as an 
electronic medical record for patients, allowing the storage of phenotypic and genotypic data through a web interface.

Specific queries were run by the data management team to identify patients with sarcoidosis in the database. For patients transferred to adult clinical pulmonary centres for further follow-up care, clinical information was obtained from the local hospital records. The patients presenting with a non-thoracic sarcoidosis, a Blau syndrome, an immune disorder, or a Crohn disease, were excluded. All included patients had a histologic diagnosis of sarcoidosis, made by a paediatric pathologist. ${ }^{14} 15$ The presence of a typical granuloma in at least one involved organ was considered compulsory for the diagnosis of sarcoidosis.

At initial evaluation the following items were collected: patient information (date of birth, gender, ethnical origin, familial consanguinity), age at diagnosis, familial history of sarcoidosis, general symptoms (fever, anorexia, asthenia and weight loss), and clinical manifestations of organ involvement. Standard blood laboratory tests included measurements of angiotensinconverting enzyme (ACE, normal range depending on the laboratory), erythrocyte sedimentation rate (ESR, normal range $<8 \mathrm{~mm} / \mathrm{h}$ ) and calcaemia (normal range 2.2-2.6 mmol L). Lung disease was evaluated by chest imaging (chest X-ray and highresolution CT (HRCT) scan), pulmonary function tests, and bronchoalveolar lavage (BAL) fluid analysis. Current HRCT scan and pulmonary function test protocols used by the RespiRare participating centres have been recently reported. ${ }^{16}$ Pulmonary function tests included measurements of lung volumes, with herein reported indices of FVC, lung transfer factor for carbon monoxide with a rebreathing method (TLCO), lung mechanics studies using the oesophageal catheter technique with measurements of dynamic lung compliance $\left(\mathrm{C}_{\mathrm{Ldyn}}\right)$, and arterial blood gas levels. The best spirometric curve out of two reproducible curves was recorded. All results were expressed as percentage of predicted values for ethnicity, gender, age and height, according to the reference values. ${ }^{17}$ FVC values $<80 \%$, and TLCO and $\mathrm{C}_{\text {Ldyn }}$ values $<75 \%$ were considered as significantly decreased. Bronchoscopy and BAL were performed as previously described, with cytological analysis of BAL fluid including the CD4/CD8 lymphocyte ratio. ${ }^{18}$

The physician in charge of the patient decided on the treatment option and performed the patient follow-up. During the follow-up all patients were evaluated at several time points, at least every 3 months during the first year of follow-up, and depending on the evolution after. The patients were considered as (1) cured: in situations of absence of an organ involvement and no sequelae of the disease in the absence of treatment; (2) stable: in situations of persistence of an organ involvement, with no worsening of the disease and no evidence for a new organ involvement; (3) experiencing relapses: in all other situations.

Quantitative values were reported as median and range or mean and SDs. Qualitative data were reported as number (percentages); associations between age at diagnosis, lung function tests, treatments and relapses were studied. Comparisons between groups were established using a non-parametric $t$ test and prognosis factors were analysed using a Mann-WhitneyWilcoxon test. p Values less than 0.05 were considered statistically significant.

\section{RESULTS}

Forty-one patients with paediatric sarcoidosis have been registered into the e-RespiRare database since 2008. They were diagnosed and followed by pulmonary paediatric teams in seven university hospitals in France. After the age of 18 years, they were referred to the adult teams to pursue the follow-up.

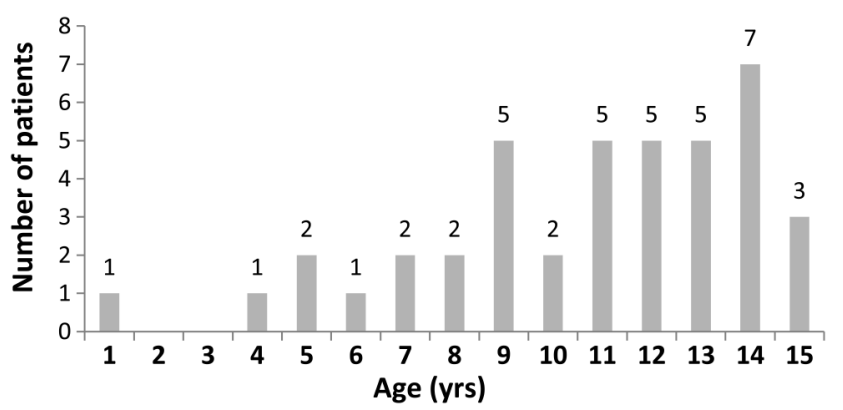

Figure 1 Age distribution in years at diagnosis of the 41 patients included in the study.

The female/male sex ratio was 1.15. Age distribution at diagnosis is shown in figure 1 . Median age at diagnosis was 11.8 years old (1.1-15.8). The median (range) duration of follow-up after disease onset was 58 months (8-183). From previous reports indicating age differences in disease expression, two groups of patients were defined in the present study: the group of patients (14 patients, 7 girls) younger than 10 years old at diagnosis ( $<10$ years group), and the group of patients (27 patients, 15 girls) 10 years old and older at diagnosis ( $\geq 10$ years group). ${ }^{12}$ Thirty-six were Afro-Caribbean or Sub-Saharan Africans, one was from India and four patients were Caucasian. A familial history of sarcoidosis was reported for five patients, including two brothers from inbreeding parents.

Clinical manifestations at diagnosis are summarised in table 1. General symptoms were observed in a large number of patients, with mainly asthenia, fever (reported in $71 \%$ patients of the $<10$ years group, and in only $29 \%$ in the $\geq 10$ years group), and weight loss.

Table 1 Clinical manifestations of the study population (41 patients) at diagnosis

\begin{tabular}{lll}
\hline & $\begin{array}{l}\text { Number of } \\
\text { patients }\end{array}$ & Percentage \\
\hline General & 32 & 78 \\
Fatigue & 23 & 56 \\
Fever & 18 & 44 \\
Weight loss & 18 & 44 \\
Sweats & 4 & 10 \\
Lung & 23 & 56 \\
Cough & 14 & 34 \\
Dyspnoea & 10 & 24 \\
Liver, spleen & 20 & 49 \\
$\quad$ Hepatomegaly and/or splenomegaly & 16 & 39 \\
Eyes & & \\
$\quad$ Uveitis, iridocyclitis, conjunctivitis & 12 & 29 \\
Peripheral lymphadenopathy & 8 & 19 \\
Skin & & \\
$\quad$ Erythema, erythema nodosum, rash, sarcoid skin & & \\
$\quad$ lesions & & \\
Joints & 6 & \\
$\quad$ Arthritis & & \\
$\quad$ ENT & & \\
$\quad$ Parotitis, salivary glands hypertrophy & 3 & 5 \\
$\quad$ Nervous system & & \\
$\quad$ Peripheral neuropathy, meningitis, migraine & 2 & 5 \\
$\quad$ Heart & & \\
$\quad$ Heart murmur & 2 & \\
\hline
\end{tabular}




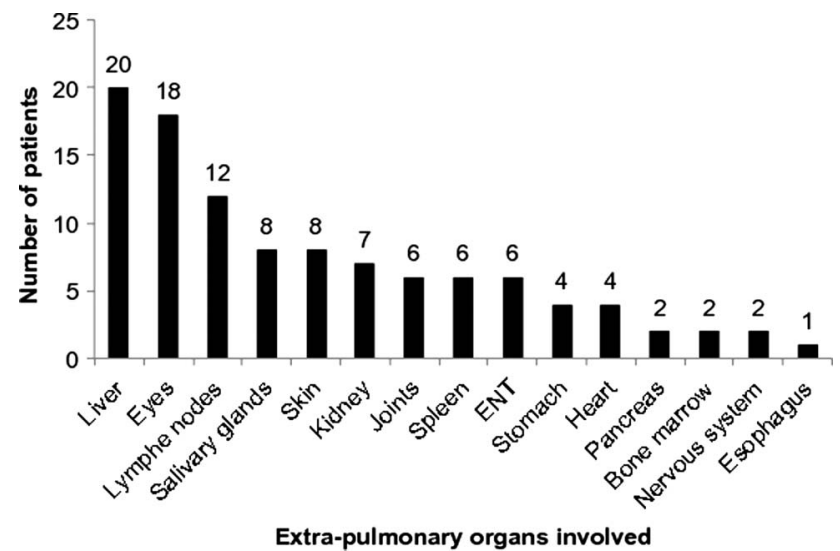

Figure 2 Extra-pulmonary organ involvement at diagnosis, assessed by extensive explorations. ENT, ear, Nose, throat.

Respiratory symptoms were documented in 23 patients. Only 6 patients had a disease limited to the respiratory system. In the other 35 patients, various combinations of organ manifestations were observed after extensive investigations (figure 2).

Chest X-rays were reported to be normal in 18 patients (44\%), but HRCT scans revealed abnormal findings in 39 patients (95\%). These abnormalities were mainly nodules with ground-glass opacities and hilo-mediastinal lymphadenopathies. Lung function tests were altered in 36 of the 38 patients who completed the tests (table 2). A significant decrease in FVC was documented in 24 patients with similar levels of alterations in the two age groups. TLCO was decreased in 20 patients, mainly in the $\geq 10$ years group. As previously described, $C_{\text {Ldyn }}$ was the functional parameter that was the most reduced in both age groups. Bronchoscopy with BAL, performed in 37 patients, documented an increase in the lymphocyte population in 35 of them, with a mean value of $40 \%( \pm 22)$; and a mean value of
CD4/CD8 ratio of $4( \pm 4)$, with a wide range from 0.28 to 19 . Results of serum ACE performed in 40 patients showed an increase in $60 \%$ patients with mean fold values of $1.5( \pm 0.8)$ the expected values. Hypercalcaemia was documented in three patients, and ESR was elevated in all but three patients with a mean value of $44 \mathrm{~mm} / \mathrm{h}( \pm 29.5)$.

As indicated in figure 2, complete clinical investigations led to liver disease documented in half of the patients. Eye lesions and enlargement of the peripheral lymph nodes were present in $44 \%$ and $29 \%$ of the patients, respectively. Involved organs in the $10-20 \%$ range of the cases include the salivary glands, skin, kidney, joints, spleen, and ear nose throat. The stomach, heart, pancreas, bone marrow, nervous system and oesophagus were rarely involved.

Altogether, a mean value of 3.8 organ involvement per patient was observed, with no statistical differences between the two groups: 3.4 in the $<10$ years group and 4 in the $\geq 10$ years group.

In more than half of the patients, several tissue specimens were needed to document the presence of typical granulomas, confirming the difficulty in establishing the diagnosis. Figure 3 indicates the sites of the tissue biopsies. As shown, the most informative sites were the salivary gland, the liver and the lung.

One patient did not receive any treatment at diagnosis. All other patients received a corticosteroid regimen, including daily oral administration of prednisolone or prednisone at doses ranging from 0.5 to $2 \mathrm{mg} / \mathrm{kg} /$ day for a median duration of 23.9 months (1-84), and/or monthly intravenous pulses of methylprednisone $\left(300 \mathrm{mg} / \mathrm{m}^{2} /\right.$ day for 3 consecutive days). At diagnosis, 17 (41\%) patients were treated with oral steroids alone, 22 (53\%) patients received intravenous pulses combined with oral steroids, and one patient was treated with intravenous pulses only.

Only one oral steroid molecule was declared for each patient with no changes over time (in half of the cases prednisolone and in the other half prednisone. The $<10$ years group received

Table 2 Patient evaluation and treatment at diagnosis, at 18 months and at 4-5 years of follow-up

\begin{tabular}{|c|c|c|c|c|c|c|}
\hline \multirow[b]{2}{*}{$\begin{array}{l}\text { Age groups and number } \\
\text { of patients ( } n)\end{array}$} & \multicolumn{2}{|l|}{ At diagnosis } & \multicolumn{2}{|c|}{ At 18 months follow-up } & \multicolumn{2}{|c|}{ At $4-5$ years of follow-up } \\
\hline & $\begin{array}{l}<10 \text { years group } \\
(14)\end{array}$ & $\begin{array}{l}\geq 10 \text { years group } \\
(27)\end{array}$ & $\begin{array}{l}<10 \text { years group } \\
(14)\end{array}$ & $\begin{array}{l}\geq 10 \text { years group } \\
(24)\end{array}$ & $\begin{array}{l}<10 \text { years group } \\
(10)\end{array}$ & $\begin{array}{l}\geq 10 \text { years group } \\
(19)\end{array}$ \\
\hline \multicolumn{7}{|l|}{ Lung function tests } \\
\hline \multicolumn{7}{|l|}{ Mean values (SD) } \\
\hline FVC \% pred & $62( \pm 26)$ & $61( \pm 22)$ & $76( \pm 12)$ & $76( \pm 15)$ & $80( \pm 12)$ & $74( \pm 13)$ \\
\hline$C_{\text {Ldyn }} \%$ pred & $45( \pm 15)$ & $43( \pm 1)$ & $66( \pm 30)$ & $65( \pm 13)$ & 100,80 and $34^{*}$ & 55 and $74^{*}$ \\
\hline TLCO \% pred & $96( \pm 48)$ & $61( \pm 19)$ & $82( \pm 12)$ & $76( \pm 21)$ & $78( \pm 8)$ & $79( \pm 23)$ \\
\hline \multicolumn{7}{|l|}{ Treatment } \\
\hline \multicolumn{7}{|l|}{ Number of patients (\%) } \\
\hline \multicolumn{7}{|l|}{ Steroids } \\
\hline Oral & $14(100 \%)$ & $23(85 \%)$ & $5(36 \%)$ & $12(50 \%)$ & $4(40 \%)$ & $8(42 \%)$ \\
\hline Intravenous pulses & 11 (79\%) & $11(41 \%) \dagger$ & $7(50 \%)$ & $4(17 \%)$ & $7(70 \%)$ & $5(26 \%)$ \\
\hline Immunosuppressive drugs & 0 & 0 & $3(21 \%)$ & $12(50 \%)$ & $3(30 \%)$ & $7(37 \%)$ \\
\hline \multicolumn{7}{|l|}{ Outcome } \\
\hline \multicolumn{7}{|l|}{ Number of patients (\%) } \\
\hline Recovery & & & $7(50 \%)$ & $7(29 \%)$ & $5(50 \%)$ & $8(42 \%)$ \\
\hline Stabilisation & & & $3(21 \%)$ & $3(13 \%)$ & $2(20 \%)$ & $2(10 \%)$ \\
\hline Relapses & & & $4(29 \%)$ & $14(58 \%) \dagger$ & $3(30 \%)$ & $9(47 \%)$ \\
\hline
\end{tabular}




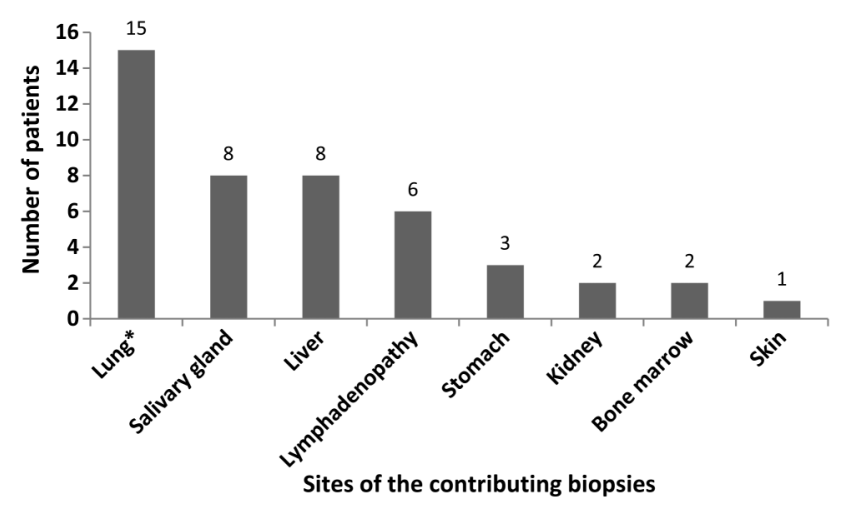

Figure 3 Sites of contributing biopsies. *Transbronchial biopsies $n=8$, surgical lung biopsy $n=7$.

significantly more intravenous pulses than the other group $(79 \%$ vs $41 \%$ ) (table 2). At diagnosis no immunosuppressive drugs were given.

Patient data were analysed after a follow-up of 18 months, and $4-5$ years. In the 38 patients who completed at least an 18-month follow-up, outcome information is summarised in table 2 . In the $<10$ years group, 7 of the 14 patients recovered completely and were free of health-related problems from sarcoidosis; 3 patients displayed clinical manifestations stabilised or improved; and 4 (29\%) experienced relapses leading to an increase in steroid treatment and for 3 of them immunosuppressive therapy was introduced. In the $\geq 10$ years group, a complete recovery was observed in 7 of the 24 patients who completed the follow-up, and a clinical improvement or stabilisation was reported in 3 children. Interestingly, relapses were more frequent in this group than in the $<10$ years group and were documented in $14(58 \%)$ patients, leading to the initiation in 12 of them of immunosuppressive therapy (mycophenolate mofetil, hydroxychloroquin, infliximab, methotrexate, azathioprine, cyclophosphamid).

A 4-5-year follow-up was completed in 29 patients, 10 in the $<10$ years group and 19 in the $\geq 10$ years group. In the $<10$ years group, only 3 patients $(30 \%)$ experienced transient relapses at 36, 54 and 56 months following the diagnosis. Two patients currently have no clinical manifestations and are receiving no treatment, and the third one has presented with multiple splenic relapses and is being treated with steroids and immunosuppressive drugs. In the $\geq 10$ years group, relapses were observed in 9 patients (47\%), with current treatment regimens including oral steroids in 8 , and intravenous steroids in 5 . Seven of these patients receive immunosuppressive drugs.

Analysis of relapse occurrence indicated that it was significantly associated with systemic disease extension, evaluated by the number of involved organs $(p=0.004)$. No other associations could be documented, such as associations with lung disease expression assessed by chest X-ray or HRCT scan abnormalities and CD4/CD8 ratio in the BAL at diagnosis, and with ACE or ESR levels.

\section{DISCUSSION}

The present study used the national institutional organization RespiRare to gather all the paediatric cases with a diagnosis of pulmonary sarcoidosis. The recruited population was composed of 41 patients, providing an accurate description of disease features, management and outcome, and allowing the results to be compared with the paediatric Danish study and with adult studies.
The composition of the present cohort is characterised by a number of cases in young children that is slightly higher than in other reports. Most of the epidemiologic data on paediatric sarcoidosis have been obtained from the Danish national patient registry. ${ }^{719}$ In this registry, established from 1979 to 1994 , and re-evaluated in 1999, age distribution analysis showed that 6 children out of $46(13 \%)$ were diagnosed before the age of 10 , compared with 14 out of $41(34 \%)$ in the present study. Most likely, this difference is not explained by changes in incidence but rather by an increased recognition of disease features and manifestations. The other main difference between the two cohorts is the racial repartition with a majority of Afro-Caribbean and Sub-Saharan patients in the present study, whereas the Danish cohort is mainly Caucasian. This could be explained by a high rate of immigration in France, with most migrants coming from southern countries. ${ }^{20}$

Clinical manifestations were marked by a high rate of general symptoms and especially fever in the youngest patients. This is infrequent in adults. ${ }^{14} 21$ This observation could be due to a revealing infection, or to a particular inflammatory phenotype of the disease in young children. Fatigue is an under-reported symptom that was at the forefront in this study. ${ }^{22-24}$ The main reported symptoms (respiratory, hepatomegaly and/or splenomegaly, and uveitis) differed from those reported in the Danish cohort, with skin lesions, iridocyclitis and peripheral lymphadenopathy. The reported clinical data are more in keeping with the adult ACCESS study, probably due to the high percentage of black patients, who are reported to have more severe and extended forms of the disease. ${ }^{10}{ }^{25-27}$ This observation emphasises the importance of detection of all possible organ localisations. This strategy allowed detection of the multi-organic extension of the disease in $85 \%$ of the children, with 3.8 localisations per patient, and no differences between the two age groups. A cardiovascular involvement (explored in 35 patients) was documented in 4 patients (conduction and rhythm disturbances).

Biological information confirms the knowledge that in children, they are not sufficient to assess the diagnosis. In this population, BAL fluid analysis showed most of the time lymphocyte overexpression, with an elevated CD4/CD8 rate, but its absence did not eliminate the diagnosis of sarcoidosis. ACE remained normal in some children, and cannot be used as a single diagnosis marker. In almost all patients, ESR was elevated, supporting the view of a specific inflammatory status in the paediatric population, as it is rarely observed in adults. Finally, hypercalcaemia was reported in three patients $(7 \%)$ versus up to $30 \%$ of the patients in the Danish cohort; this difference was not explained.

In adult sarcoidosis, therapeutic abstention can reach up to $20 \%$ of the cases for newly diagnosed sarcoidosis. ${ }^{28-30}$ However, here, it was the case for only one patient. Moreover, systemic corticosteroids were prescribed as intravenous pulses and/or with an initial high regimen of oral corticosteroid, up to twice the recommended adult dose and duration of treatment. These differences between adult and paediatric management could be due to the severe forms of sarcoidosis described here, with multi-organ involvement, in these patients mainly from Afro-Caribbean and Sub-Saharan origin. ${ }^{12}$ In children, in the absence of systematic chest X-rays, mild sarcoidosis may be under-diagnosed in children.

In this paediatric study, we found no differences in disease severity at presentation, regardless of patient age at diagnosis. The short-term outcome ( 18 months) is marked by a low rate of healing: only a third of the patients recovered (13/38 patients, $34 \%)$. Children diagnosed before the age of 10 years old 
received an aggressive treatment with mostly intravenous steroid pulses. Interestingly, these younger patients presented with fewer relapses before 18 months of follow-up than the oldest patients. The patients who received immunosuppressive drugs as a second intention treatment (15 patients) might be considered as non-responders to steroids, with no influence of the type of oral steroid molecules. It is important to note that the patients who were started on steroid pulses at diagnosis required fewer immunosuppressive drugs during the follow-up, suggesting that an initial high steroid regimen may be more efficient in preventing long-term relapses or worsening.

The long-term outcome (4-5 years) seems to be less favourable than described in the Danish cohort. It could be performed only for $70 \%$ of the patients (29/41). In both age groups, only half of the patients had recovered at that time, mostly without any sequelae, as it was also described in adults by Milman et al. ${ }^{31}$ Long-term relapses were more frequently reported in the $\geq 10$ years group, and mainly of extra-thoracic localisation. It should be pointed out that there is currently no standardised definition of relapses. Recurrence of the disease may be considered as an acute exacerbation. The disease may also have never been controlled but only attenuated by the treatment. More work is needed to define criteria of exacerbations and relapses in paediatric sarcoidosis and other forms of interstitial lung diseases. ${ }^{32} 33$

The present study provides information on a national experience in paediatric sarcoidosis. It displays, however, some limitations that are mainly related to the lack of exhaustivity, especially for the mild cases. Disease prevalence and incidence being major issues of the RespiRare network, a work organisation has been developed to facilitate case implementation and, therefore, to identify most of the patients with specific rare lung diseases. The dedicated effort for continued and improved surveillance of paediatric sarcoidosis occurrence will provide reliable epidemiologic data in the near future. Currently, based on the number of new cases diagnosed in France, a rough estimated incidence of $0.4-0.8$ per 100000 children could be suggested.

This study points out the severity of sarcoidosis in children. This French cohort is found to be mostly of Afro-Caribbean and Sub-Saharan origin, with a multi-organic involvement and foreground general symptoms, probably because of specific genetic and environmental modifiers factors. Common prognosis factors of sarcoidosis are not suitable in paediatric patients and the only parameter that we found to be associated with relapse occurrence is the number of involved organs. Contrary to what is usually believed, no differences were found in disease expression at presentation between patients diagnosed before or after 10 years old. In addition a young age at diagnosis does not seem to be associated with a poorer prognosis. The follow-up observation that the $<10$ years group experienced fewer relapses (although similar severity) than the older group may be explained by the more aggressive treatment at presentation. The study is ongoing within the network of RespiRare and adult reference centres, with the aim to collect further information on a very-long term outcome of the disease, to progress in the phenotypic description, and to improve the therapeutic strategies.

\footnotetext{
Author affiliations

${ }^{1}$ Pediatric Pulmonary Department, AP-HP, Hôpital Trousseau, Paris, France

${ }^{2}$ Université Pierre et Marie Curie-Paris6, Paris, France

${ }^{3}$ UMR S-U933, Inserm, Paris, France

${ }^{4}$ Pediatric Department, Centre Hospitalier Universitaire de Lille, Lille, France

${ }^{5}$ Pediatric Pulmonary Department, AP-HP, Hôpital Robert Debré, Paris, France

${ }^{6}$ Pediatric Department, Centre Hospitalier Intercommunal de Créteil, Créteil, France
}

${ }^{7}$ U955, Equipe 11, Inserm, Créteil, France

${ }^{8}$ Faculté de Médecine, Université Paris-Est, Créteil, France

${ }^{9}$ Pediatric Pulmonary Department, AP-HP, Hôpital Necker Enfants Malades, Paris, France

${ }^{10}$ Université Paris Descartes-Paris5, Paris, France

${ }^{11}$ AP-HP, Hôpital Avicenne, Pulmonary Department and I'Université Paris 13 , COMUE Sorbonne Paris Cité, EA 2363, Bobigny, France

${ }^{12}$ Pediatric Department, Centre Hospitalier Universitaire de Dijon, Dijon, France

${ }^{13}$ UMR S-707, Inserm, Paris, France

Acknowledgements The authors want to thank all the patients, the paediatric clinicians of RespiRare for providing information on their patients, the adult Respifil network for ensuring the long-term follow-up of the paediatric patients when they moved to adult clinics, and the French Sarcoidosis Group.

Contributors $N N, V H, R E, J d B, D V, A H, H C, A D$, and $A C$ were in charge of study design, patient inclusion, selection and implementation of phenotypic data and manuscript review. NN and AC wrote and finalised the manuscript.

Funding The research leading to these results received funding from: Legs Poix, grant no 1305 and no 1405, and from the European Union's Seventh Framework Program (FP7-ChILD-EU 2007-2013) under grant agreement no 305653.

Competing interests None declared.

Ethics approval CCTIRS no 08.015bis, CNIL.

Provenance and peer review Not commissioned; internally peer reviewed.

Data sharing statement The authors give to Thorax their acceptance for data sharing.

\section{REFERENCES}

1 Hunninghake GW, Costabel U, Ando M, et al. ATS/ERS/WASOG statement on sarcoidosis. American Thoracic Society/European Respiratory Society/World Association of Sarcoidosis and other Granulomatous Disorders. Sarcoidosis Vasc Diffuse Lung Dis 1999;16:149-73.

2 lannuzzi MC, lyengar SK, Gray-McGuire C, et al. Genome-wide search for sarcoidosis susceptibility genes in African Americans. Genes Immun 2005;6:509-18.

3 Iannuzzi MC, Rybicki BA, Teirstein AS. Sarcoidosis. N Engl J Med 2007;357:2153-65.

4 Spagnolo P, Grunewald J. Recent advances in the genetics of sarcoidosis. J Med Genet 2013;50:290-7

5 Dubrey S, Shah S, Hardman T, et al. Sarcoidosis: the links between epidemiology and aetiology. Postgrad Med J 2014;90:582-9.

6 Byg K-E, Milman N, Hansen S. Sarcoidosis in Denmark 1980-1994. A registry-based incidence study comprising 5536 patients. Sarcoidosis Vasc Diffuse Lung Dis 2003;20:46-52.

7 Hoffmann AL, Milman N, Byg KE. Childhood sarcoidosis in Denmark 1979-1994: incidence, clinical features and laboratory results at presentation in 48 children. Acta Paediatr 2004;93:30-6.

8 Rybicki BA, lannuzzi MC, Frederick MM, et al. Familial aggregation of sarcoidosis. A case-control etiologic study of sarcoidosis (ACCESS). Am J Respir Crit Care Med 2001;164:2085-91.

9 Judson MA, Boan AD, Lackland DT. The clinical course of sarcoidosis: presentation, diagnosis, and treatment in a large white and black cohort in the United States. Sarcoidosis Vasc Diffuse Lung Dis 2012;29:119-27.

10 Baughman RP, Teirstein AS, Judson MA, et al. Clinical characteristics of patients in a case control study of sarcoidosis. Am J Respir Crit Care Med 2001;164:1885-9.

11 Nunes H, Soler P, Valeyre D. Pulmonary sarcoidosis. Allergy 2005;60:565-82.

12 Baculard A, Blanc N, Boule $M$, et al. Pulmonary sarcoidosis in children: a follow-up study. Eur Respir J 2001;17:628-35.

13 Nathan N, Abou Taam R, Epaud R, et al. A national internet-linked based database for pediatric interstitial lung diseases: the French network. Orphanet J Rare Dis 2012;7:40.

14 Valeyre D, Bernaudin J-F, Uzunhan Y, et al. Clinical presentation of sarcoidosis and diagnostic work-up. Semin Respir Crit Care Med 2014;35:336-51.

15 Heinle R, Chang C. Diagnostic criteria for sarcoidosis. Autoimmun Rev 2014;13:383-7.

16 Sileo C, Epaud R, Mahloul M, et al. Sarcoidosis in children: HRCT findings and correlation with pulmonary function tests. Pediatr Pulmonol 2014;49:1223-33.

17 Hankinson JL, Odencrantz JR, Fedan KB. Spirometric reference values from a sample of the general U.S. population. Am J Respir Crit Care Med 1999;159:179-87.

18 Chadelat K, Baculard A, Grimfeld A, et al. Pulmonary sarcoidosis in children: serial evaluation of bronchoalveolar lavage cells during corticosteroid treatment. Pediatr Pulmonol 1993; 16:41-7.

19 Milman N, Hoffmann AL. Childhood sarcoidosis: long-term follow-up. Eur Respir J 2008;31:592-8

20 Rybicki BA, Major M, Popovich J, et al. Racial differences in sarcoidosis incidence: a 5-year study in a health maintenance organization. Am J Epidemio/ 1997;145:234-41.

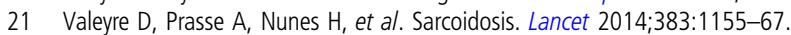

22 De Kleijn WPE, De Vries J, Lower EE, et al. Fatigue in sarcoidosis: a systematic review. Curr Opin Pulm Med 2009;15:499-506. 


\section{Paediatric lung disease}

23 Baughman RP, Nunes H. Sarcoidosis-associated fatigue: an often forgotten symptom - author reply. Expert Rev Clin Immunol 2013;9:111.

24 Kapoor S. Sarcoidosis-associated fatigue: an often forgotten symptom. Expert Rev Clin Immunol 2013:9:109-10.

25 Judson MA, Costabel U, Drent M, et al. The WASOG Sarcoidosis Organ Assessment Instrument: an update of a previous clinical tool. Sarcoidosis Vasc Diffuse Lung Dis 2014;31:19-27.

26 Judson MA. The clinical features of sarcoidosis: a comprehensive review. Clin Rev Allergy Immunol. Published Online First: 2 Oct 2014. doi:10.1007/s12016-0148450-y

27 Evans M, Sharma O, LaBree L, et al. Differences in clinical findings between Caucasians and African Americans with biopsy-proven sarcoidosis. Ophthalmology 2007;114:325-33.
28 Gibson GJ. Sarcoidosis: old and new treatments. Thorax 2001;56:336-9.

29 Londner C, Zendah I, Freynet 0, et al. [Treatment of sarcoidosis]. Rev Med Interne 2011;32:109-13.

30 Baughman RP, Lower EE. Medical therapy of sarcoidosis. Semin Respir Crit Care Med 2014;35:391-406.

31 Milman N, Svendsen CB, Hoffmann AL. Health-related quality of life in adult survivors of childhood sarcoidosis. Respir Med 2009;103: 913-8.

32 Baughman RP, Judson MA. Relapses of sarcoidosis: what are they and can we predict who will get them? Eur Respir J 2014;43:337-9.

33 Baughman RP, Lower EE. Features of sarcoidosis associated with chronic disease. Sarcoidosis Vasc Diffuse Lung Dis 2015;31:275-81. 